\title{
Application of the Positron Lifetime Spectroscopy as Method of Non-Destructive Testing
}

\author{
B. Somieski*, R. Krause-Rehberg, H. Salz and N. Meyendorf*
}

Martin-Luther-Universität Halle/Wittenberg, Fachbereich Physik, Positronenlabor, F. -Bach-Platz 6, 06108 Halle/Saale, Germany

* Fraunhofer-Institut für zerstörungsfreie Prüfverfahren, Abt. EADQ, Gruppe phys. Verfahren Universität, Gebäude 37, 66123 Saarbrücken, Germany

\begin{abstract}
In order to show the suitability of the Positron Lifetime Spectroscopy (POLIS) as a method of Non-Destructive Testing (NDT) several iron alloys / steels were mechanically damaged (tensile stress, fatigue, creeping). The positron annihilation parameters show clear changes during all applied kinds of damage. After tensile stress as well as after creeping a homogen eous distribution of damage in the sample was detected. During the very first elastic cycle of a fatigue experiment, a change in the defect structure occurs in well annealed materials.
\end{abstract}

A modified spectrometer for in the field mapping is presented.

\section{Introduction}

The investigation of the damage state of a material is very important for its industrial applications. Most mechanical damages start with a change in the microstructure of the material. In spite of its sensitivity for defects, to this day the positron annihilation technique is relatively seldom used for Non-Destructive Testing. Applications of the Positron Lifetime Spectroscopy for NDT are still not known. The POLIS has the great benefit to be able to detect microscopic defects like vacancies, dislocations or vacancy clusters "directly", which is hardly possible with other suitable methods of NDT. In addition, it is possible to distinguish between several kinds of defects.

In opposite to earlier attempts [1,2], where a positron annihilation lineshape spectrometer was used, we show the suitability of the POLIS as a method for NDT. For that reason we analysed steel stressed in different ways (tensile stress, cold-rolling, fatigue, creeping). The POLIS often shows changes in the PA-parameters prior to changes in the mechanical properties, which are detectable by other methods of NDT.

\section{Experimental}

$15 \mu \mathrm{Ci}{ }^{22} \mathrm{Na}$ acetate was used as positron source, deposited on an aluminium foil of $2 \mu \mathrm{m}$ thickness. This source was covered with the same type of aluminium foil. 
In the presented work, only one single specimen instead of two identical samples was available for the investigations. Therefore, we used a reference material exhibiting a single positron lifetime to complete the sandwich. These sandwiches were analysed in a positron lifetime spectrometer with a time resolution of about $250 \mathrm{ps}$ (full width of half maximum).

By comparing the lifetime spectra of normal source-sample setups of wellknown iron or steels with sandwiches that consist of this reference sample and the steel we were able to measure the fraction of positrons annihilating in the reference material. For GaAs (Zn-doped) $\left(\bar{\tau}=\tau_{\mathrm{b}}=\right.$ $230 \mathrm{ps}$ ) used as reference we found that $44 \%$ of the positrons annihilate in this half of the sandwich. This part of the accumulated spectra was subtracted before the decomposition was made.

In order to be able to study large specimens we modified our spectrometer by using slanting scintillators and placed the photomultipliers right-angle to each other (Figure 1). The positron source was put with a reference material piece onto the
surface of the specimen. After first tests

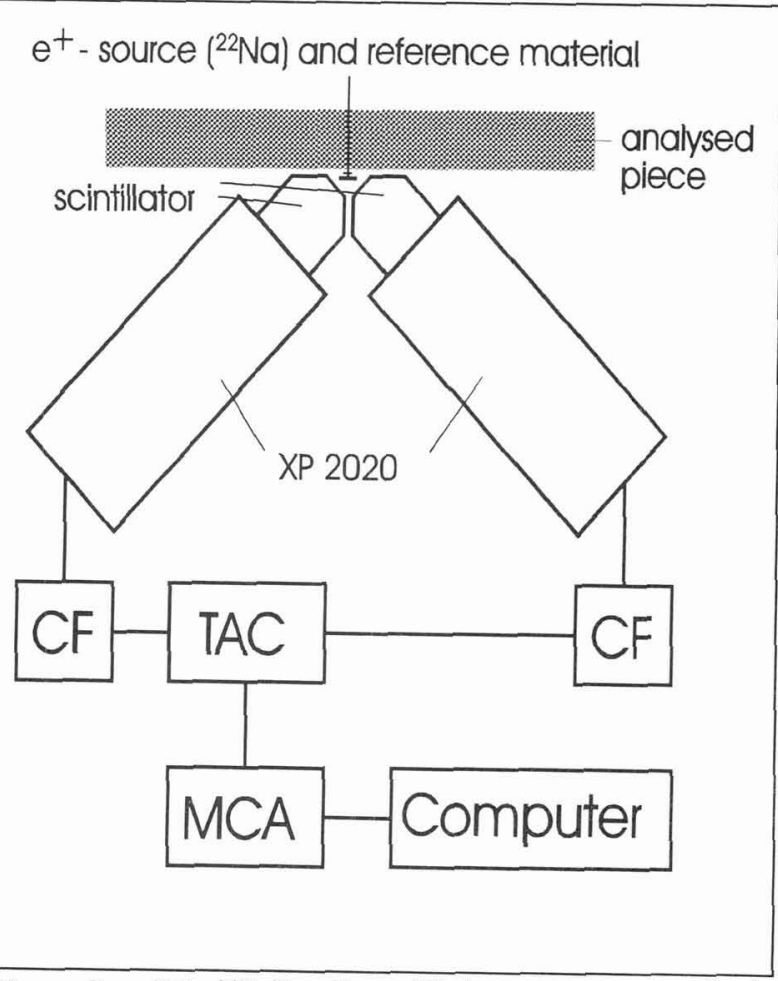
Figure 1 Modified positron lifetime spectrometer for inthe-field mapping. we found that the results from this modified spectrometer and the results from collinear photomultipliers are the same. So we are able to make in-the-field mapping of POLIS.

\section{Results and Discussion}

\subsection{Iron Alloys after tensile strain}

We investigated seven well-annealed iron alloys or steels after tensile stress. We recorded the stress-strain curve step by step on flat tensile test sheets, made according to the ISO normative ISO/R 1555-1971 (DIN 50114). The test sheets look like in Figure 4. After every step a positron lifetime spectrum was collected on the not-stressed sample. Thus, we detected only the permanent damage.

The positron annibilation spectra were analysed following the trapping model of SEEGER [3]. The increase in the average positron lifetime $\bar{\tau}$ observed is due to positron trapping to dislocations and small vacancy clusters as well. Further details of the data and more detailed discussion of the results are to be published elsewhere.

First of all, we investigated pure iron. In Figure 2 the stress-strain diagram and the average positron lifetime versus the strain are plotted together. Both plots look alike. It is important that the most significant change in the average positron lifetime occurs up to $10 \%$ of tensile strain.

To study the damage distribution in homogeneous samples, two line-scans were measured crosswise on the pure iron sample, which was successively exposed to different tensile stresses (Figure 4). Within the measuring accuracy, after all experimental steps the defect structure was absolutely homogeneously distributed except one region, where the homogeneity was disturbed at $50 \%$ 
tensile stress. In this region the sample was evidently more constricted than in the other regions, i.e. the damage in this region was higher than everywhere else. Finally, the sample cracked here. Previously, no extra constriction was visible.

In Figure 3 the average positron lifetimes of all investigated samples are plotted in a common diagram. The abscissa is normalized to the same fractions of tensile strain.

In all materials, the most rapid increase of the average positron lifetime occurs from the not strained state to $10 \%$ of tensile stress, meaning that the positron lifetime spectroscopy is particularly sensitive for the early state of damage of a material. All positron lifetime curves, drawn versus the strain, look very similar. Obviously, there is no significant difference between the materials, which is an important advantage in applying this method especially for NDT.

Until now it is not clearly understood why the average positron lifetime decreases from $50 \%$ tensile strain to the crack strain.

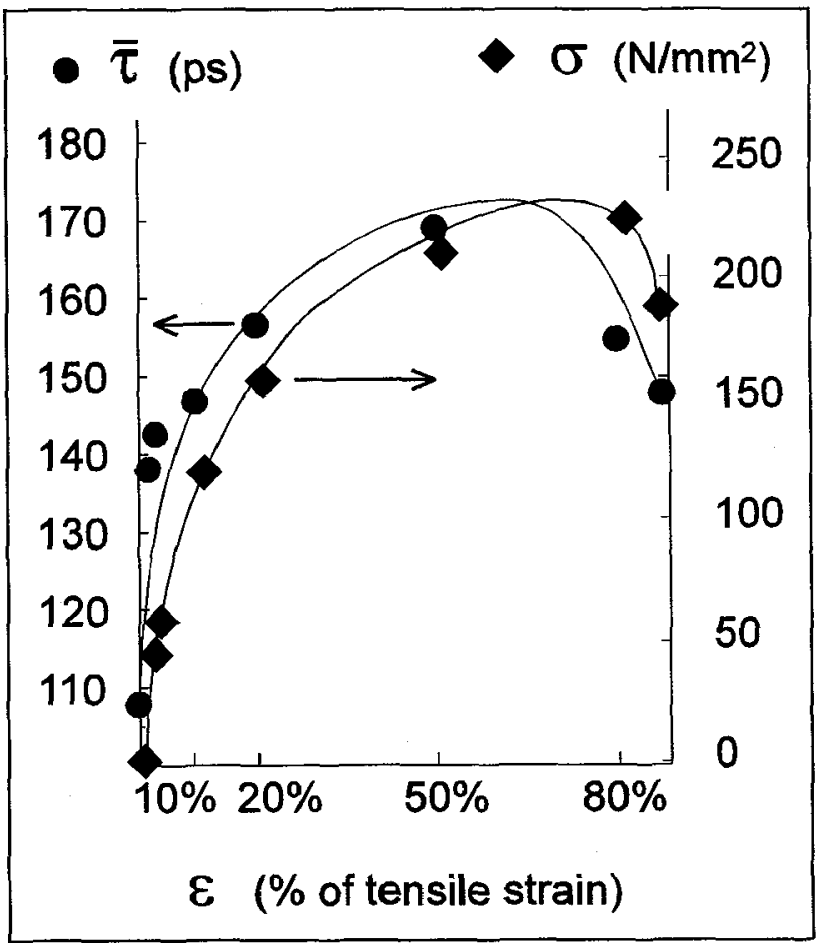

Figure 2 Average Positron lifetime in pure iron after tensile strain and stress-strain diagramm of pure iron. It seems to be possible that the dislocations join to networks and thereby form regions of low dislocation density and therefore, a lower positron lifetime is observed.

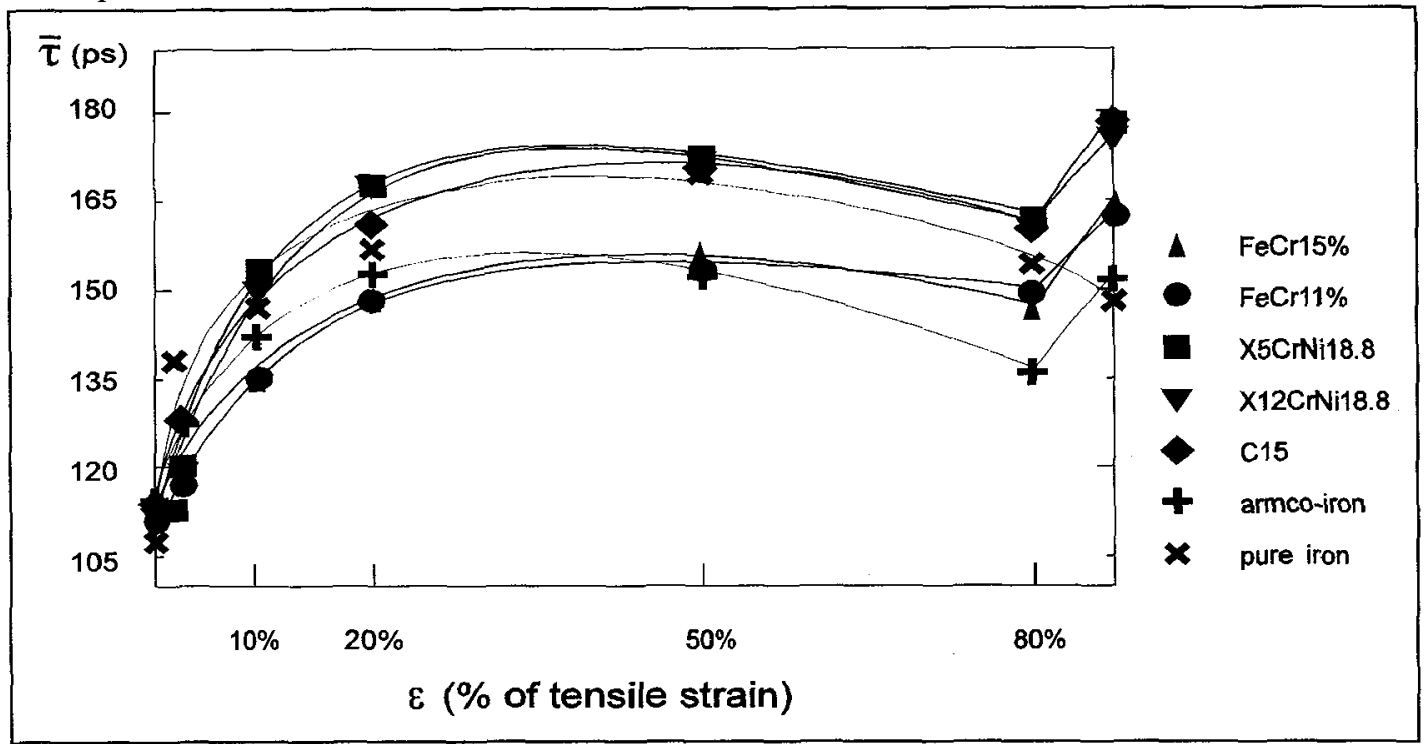

Figure 3 Average positron lifetime vs. tensile strain in iron alloys. 


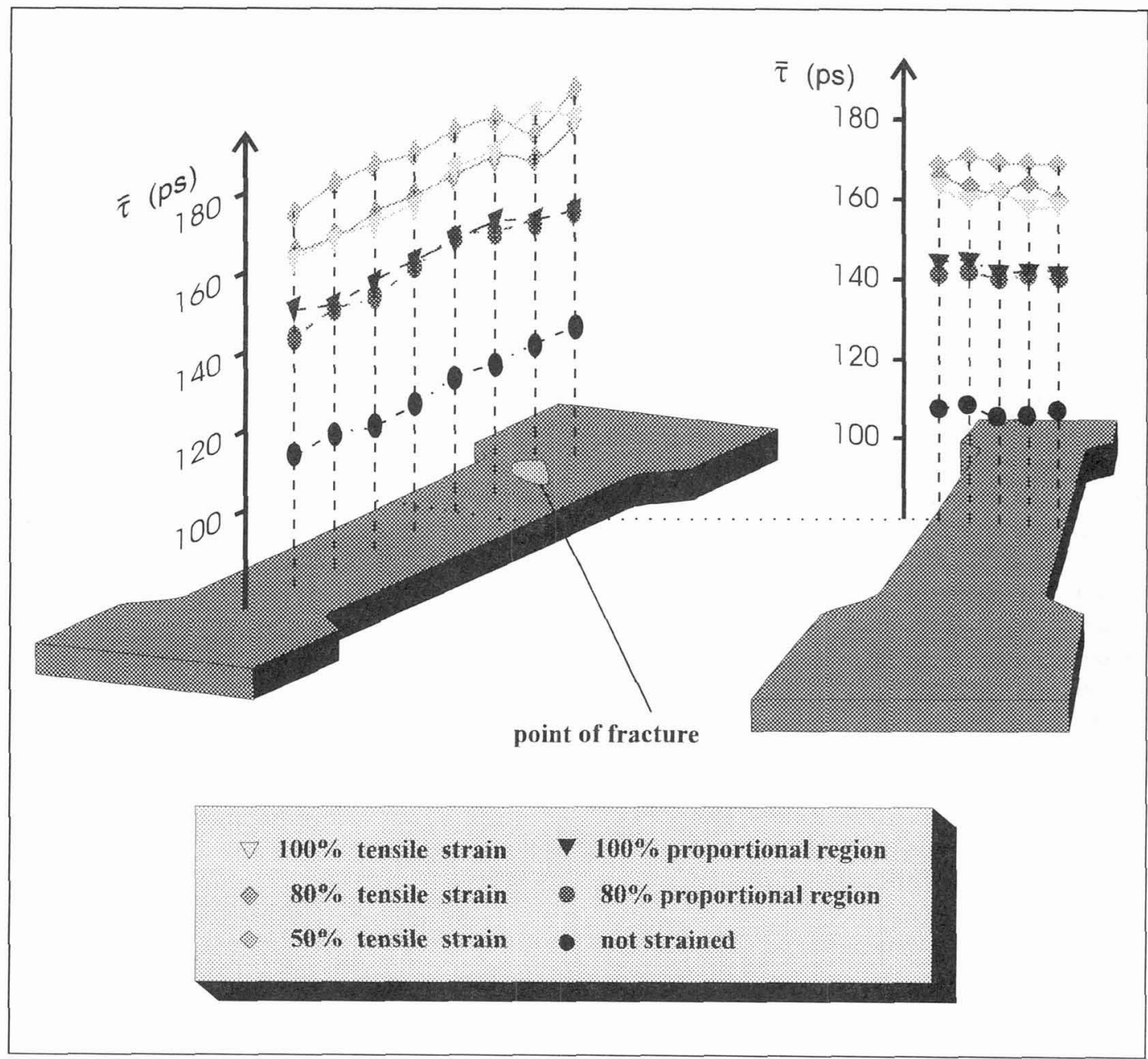

Figure 4 Distribution of the avarage positron lifetime in pure iron after tensile strain.

\subsection{Pure Imon Under Fatigue Test}

Four identical pure iron samples were fatigued with different maximal-stress amplitudes. 20, 40, 60 and $80 \%$ of the proportional region were applied. Since the samples were flat test sheets we applied a sinusoidal stress without pressure. The samples were investigated with a reference material as described above (see Figure 5).

Already after the first elastic cycle the average positron lifetime was increased in all four samples. Thus, we conclude that the samples were immediately damaged. The samples loaded with 20,40 , $60 \%$ show a weak but distinct increase in the average positron lifetime.

In the sample with a load of $80 \%$ of the proportional region the average positron lifetime increased continuously up to 100 cycles, thereafter the average positron lifetime did not increase any more up to 1000 cycles.

This type of experiment will be continued. 


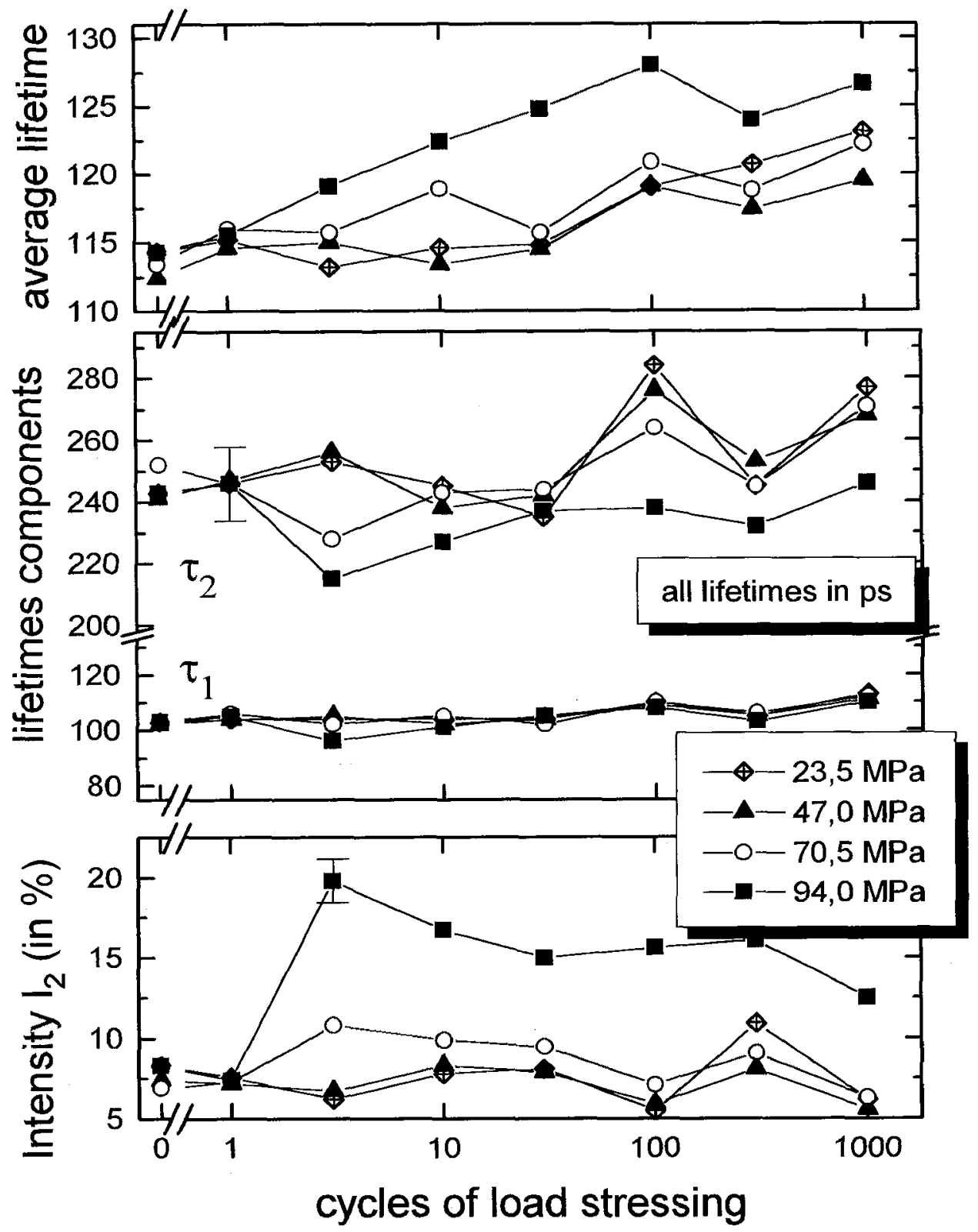

Figure 5 Positron annihilation parameters of pure iron under fatigue test. 


\subsection{Steel Under Creeping}

Samples made of the steel type 14MoV63 were loaded with $130 \mathrm{MPa}$ tensile stress and were annealed at $550^{\circ} \mathrm{C}$. A set of five samples with different creeping times were studied (Figure 6). The relative lengthening of the samples was linear to the creeping time.

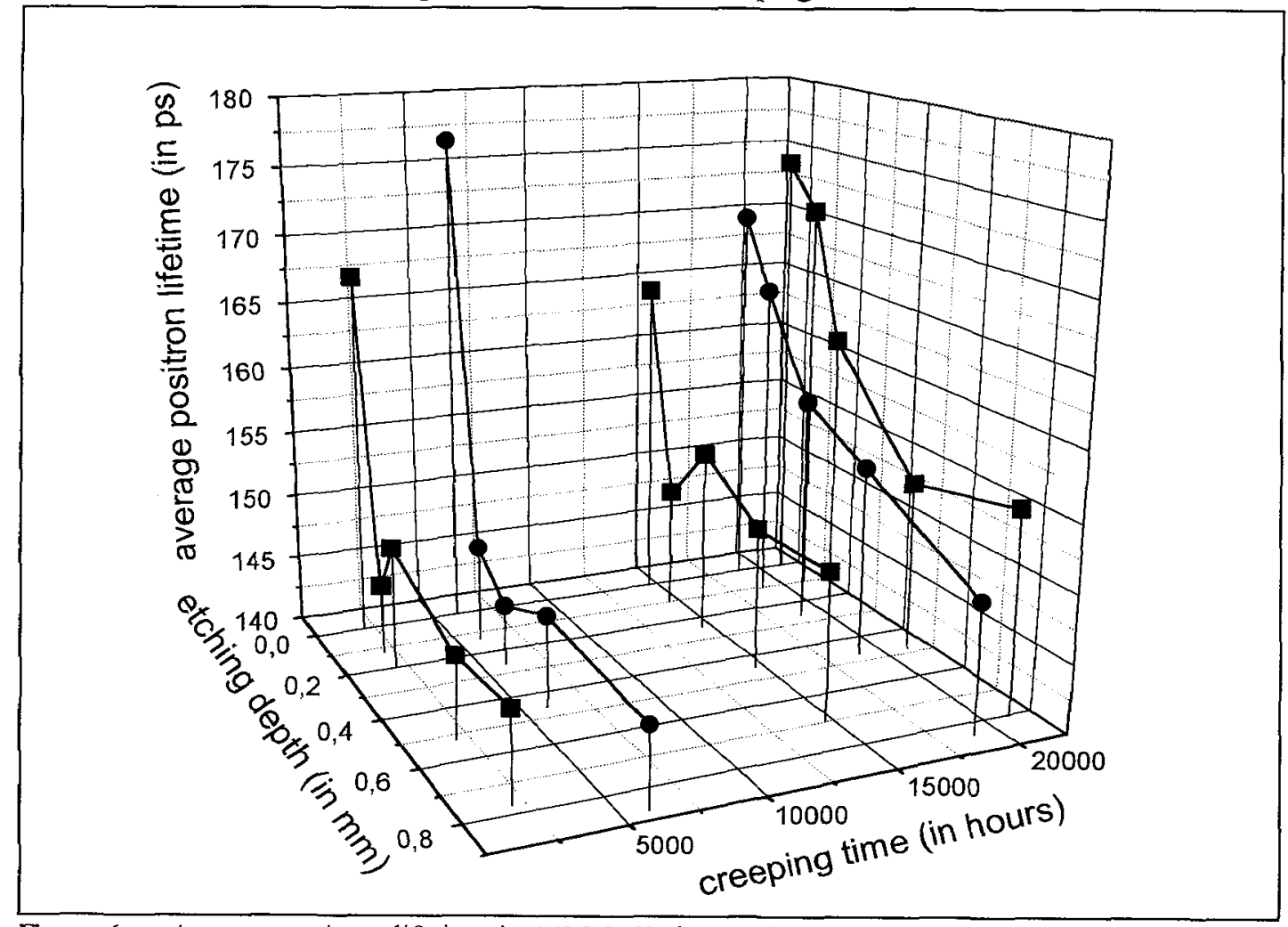

Figure 6 Average positron lifetime in $14 \mathrm{MoV} 63$ damaged by creeping $\left(130 \mathrm{MPa}, 550^{\circ} \mathrm{C}\right)$

For previous ultrasonic measurements the surface was grinded. This produced a clearly damaged surface region. For excluding this effect of grinding the samples had to be etched. After chemical etching the surface is often very rough, which can damage or destroy the positron source. In order to avoid this problem we etched the samples electrochemically, producing a quite smooth surface. Corresponding to [4] the damaged surface region was removed after the first etching step of about $0.1 \mathrm{~mm}$. This was checked by etch profiles performed at reference samples that were not treated by damage. This fact can also be found by observing the first and the second sample. There the average positron lifetime was relative constant during the whole etching procedure. That means that in all samples after the first etching step the defect structure of the material is only influenced by creeping, no more by grinding.

In the samples that were damaged by longer creeping times we found a clear gradient of $\bar{\tau}$ from the surface to the interior of the sample. In addition the value of the average lifetime in the deeper regions is higher than in the first samples. That provides the conclusion that creeping damages the surface more than the inner part of the samples. For the industrial application of the POLIS it is interesting that the most remarkable changes in the positron parameters from sample to sample occur near the surface.

In a set of five samples loaded with higher tensile stress (Figure 7) the rise of the average positron lifetime is detectable after less creeping time. But the gradient of the average lifetime from the 


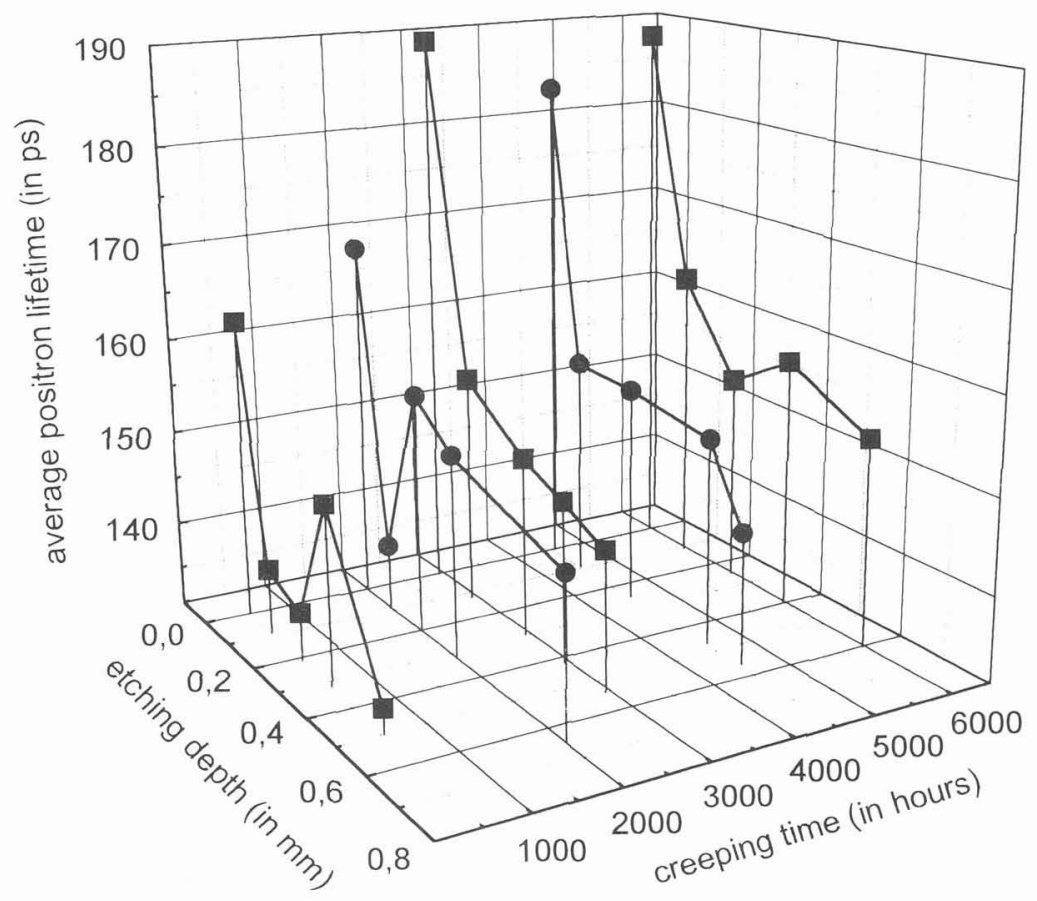

Figure 7 Average positron lifetime in $14 \mathrm{MoV} 63$ after creeping damage (165 $\mathrm{MPa}, 550^{\circ} \mathrm{C}$ ) surface to the inner part of the sample is not so evident.

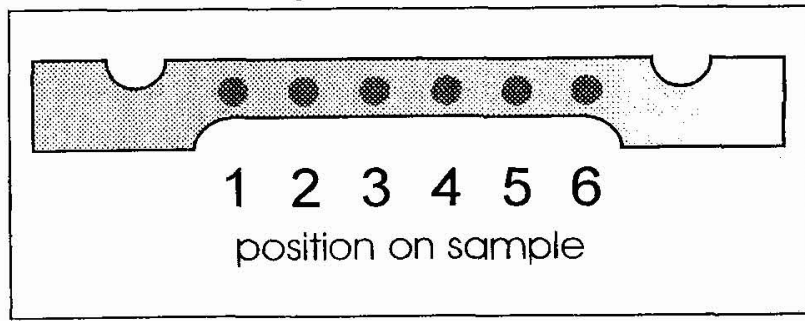

Figure 8 Sample for creeping damage.
We analysed a line scan along the surface of one sample (165 MPa, 5484h) during etching shown in Figure 8.

We found a quite homogeneous distribution of the average positron lifetime after the first and the second etching step (Figure 9). So we believe, that the damage state depends only on the depth position and not on the position on the surface. 


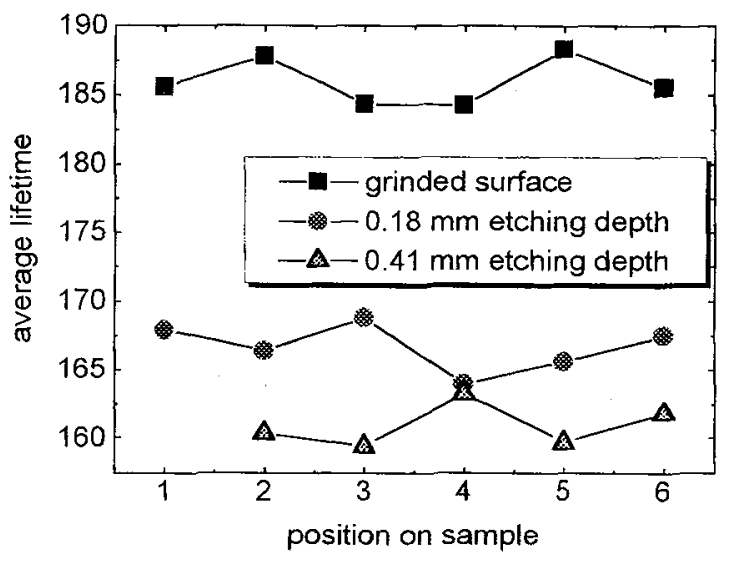

Figure 9 Distribution of the average positron lifetime after etching in the sample $14 \mathrm{MoV} 63$ (165 $\left.\mathrm{MPa}, 550^{\circ} \mathrm{C}, 5484 \mathrm{~h}\right)$.

\section{Summary}

Iron alloys or steels were loaded with several mechanical stresses. All applied damages changed the microdefect structure of the investigated samples.

In the decomposition of the lifetime spectra we found in all cases a second lifetime of about 220270 ps. We attribute this component to positron trapping in vacancy clusters. The curve shape of the average positron lifetime plotted versus the strain look alike in different iron alloys. The most significant changes occurred up to a strain of $10 \%$ temsile strain. In fatigued pure iron the $\bar{\tau}$ is already increased after the first elastic cycle. In steels samples after creeping a slope of the average lifetime was detectable, meaning that the surface region was evidently more damaged.

\section{Acknowledgements:}

The stress-strain experiments were made by T. Anhofer during his study at the "Institut für Werkstofftechnik und -prüfung der TU Magdeburg" under supervising of Dr. N. Meyendorf.

The work was supported by the "Ministerium für Wissenschaft und Forschung des Landes Sachsen-Anhalt".

\section{References:}

[1] A.J. Allen, C.F. Colman, S.J. Conchie, M.T. Hutchings, F.A. Smith, Proc. of the 4th Europ.Conf. of NDT, London UK 1987, Pergamon Press Oxford, 2193

[2] C.B. Scruby, R. Colbrook, Brit. J. of NDT 34/3 (1992) 109

[3] A. Seeger, Appl. Physics 4 (1974) 183

[4] Y.K. Park, J.T. Waber, C.L. Snead Jr., materials lett. 3/5,6 (1985) 181 